\title{
A topological charge of black holes
}

\author{
Yu $\operatorname{Tian}^{*}$ \\ School of Physics, University of Chinese Academy of Sciences, Beijing 100049, China \\ Center for Gravitation and Cosmology, College of Physical Science and Technology, \\ Yangzhou University, Yangzhou 225009, China and \\ Institute of Theoretical Physics, Chinese Academy of Sciences, Beijing 100190, China
}

(Dated: November 5, 2019)

The topological charge of a maximally symmetric black hole naturally arises in holography, which can be viewed as the last charge of the black hole in the sense that it together with all other known charges satisfies the holographic Gibbs-Duhem-like relation as a completeness relation. It is then observed that this topological charge, which we have missed before, fits perfectly into the black hole thermodynamics from two different approaches, not only in Einstein's gravity, but also in the general Lovelock-Maxwell theory.

Keywords: black hole thermodynamics; holography; topological charge; gauge/gravity 


\section{INTRODUCTION}

Black hole physics is one of the everlasting and most interesting topics in gravitation and related fields. On one hand, black holes are so simple objects that stationary black holes can be described by a small number of macroscopic parameters, which we have learned from various forms of no-hair theorems, and based on which we have black hole thermodynamics. On the other hand, black holes are so complicated that there are exceedingly rich phenomena and problems related to them, such as Hawking radiation and information loss paradox, Bekenstein-Hawking entropy and its (possible) statistical origin, Penrose process and super-radiance, black hole perturbations and quasi-normal modes, coalescence of binary black holes, hairy black holes, black hole phase transitions, black holes in AdS/CFT and holography, etc.

In this paper, we focus on the simple face of black holes. For specification and briefness, we only investigate in detail the maximally symmetric black holes in the Einstein-Maxwell theory, leaving more general cases in the Discussion. As is well known, the maximally symmetric black hole solutions in the Einstein-Maxwell theory are

$$
\begin{aligned}
d s_{d+1}^{2} & =\frac{d r^{2}}{f(r)}-f(r) d t^{2}+r^{2} d \Omega_{d-1}^{(k) 2}, \\
f(r) & =k+\frac{r^{2}}{\ell^{2}}-\frac{2 M}{r^{d-2}}+\frac{Q^{2}}{r^{2 d-4}}, \\
d \Omega_{d-1}^{(k) 2} & =\hat{g}_{i j}^{(k)}(x) d x^{i} d x^{j}, \\
A & \sim-\frac{Q}{r^{d-2}} d t,
\end{aligned}
$$

where $\ell$ is the AdS radius (related to the cosmological constant) and $k$ stands for the spatial curvature of the black hole. Specifically, $k>0, k=0$ and $k<0$ give a spherical, planar and hyperbolic geometry, respectively, and one usually takes $k=1,0,-1$ for convenience. Here and hereafter we use " $\sim$ " to denote equality up to some unimportant constant factor. It follows the first law of black hole thermodynamics

$$
d \tilde{M}=T_{H} d S+\Phi d Q
$$

with

$$
\begin{aligned}
S & =\frac{\Omega_{d-1}^{(k)} r_{h}^{d-1}}{4}, \\
T_{H} & =\frac{f_{h}^{\prime}}{4 \pi}, \quad f_{h}^{\prime}:=f^{\prime}\left(r_{h}\right), \\
\tilde{M} & \sim M, \quad \Phi \sim Q / r_{h}^{d-2} .
\end{aligned}
$$

Here $\Omega_{d-1}^{(k=1,0,-1)}$ is the volume of the "unit" sphere, plane or hyperbola, where for the latter two (noncompact) spaces certain compactifications are understood 1 Then the most important question we are going to answer is: Are there any more charges of the RN-AdS black hole (11), besides the known ones (the mass $M$ and the electric charge $Q$ ) :2

Since the above question eventually leads to an extension of the usual black hole thermodynamics, before trying to answer that question, we should review some other efforts to extend the black hole thermodynamics. The most studied case is to consider the cosmological constant $\Lambda$ as an additional thermodynamic quantity, associated with the so-called thermodynamic volume $\mathcal{V}$ as its conjugate, sometimes called the extended phase space approach (see, e.g. [1-3] ). This approach has been further generalized to modified gravitational theories (see e.g. [4] for a generalization to Lovelock gravity). These previous efforts are different from our discussion in that $\mathcal{V}$ (or other quantities conjugate to the Lovelock coupling parameters) is not a (conserved) charge, while we are focusing on all possible charges, as stated above. At the same time, considering $\Lambda$ or other coupling parameters as thermodynamic quantities goes beyond the standard thermodynamics, because they are parameters of the theory itself instead of parameters characterizing different solutions (states) in a fixed theory (system). Although some modifications of the standard Einstein(-Maxwell) gravity are known to treat $\Lambda$ as a parameter of solutions (see, e.g. [5]), it is still not clear whether full holographic frameworks exist for such kind of modified theories, which is also one of our main concerns in this paper, because we want to have a holographic interpretation of our thermodynamics (with the proposed new charge) as the real, standard thermodynamics of the dual boundary system.

\footnotetext{
${ }^{1}$ For non-standard values of $k$, the sphere or hyperbola is rescaled, so is its volume $\Omega_{d-1}^{(k)}$. See the discussions in the next section.

2 The entropy $S$ can be thought of as a Noether charge[ [6]. One more possible charge is the angular momentum $J$, as will be mentioned in the Discussion.
} 
Table I.

\begin{tabular}{|c|c|c|}
\hline Fields & Bulk & Boundary \\
\hline \hline Maxwell $A_{\mu}$ & $-\left.n_{\mu} F^{\mu a}\right|_{\text {bdry }}$ & Current $\left\langle j^{a}\right\rangle$ \\
\hline Gravitational $g_{\mu \nu}$ & Brown-York tensor $\left.t^{a b}\right|_{\text {bdry }}$ & Stress tensor $\left\langle t^{a b}\right\rangle$ \\
\hline & Local Hawking temperature & Temperature \\
\hline & Bekenstein-Hawking entropy & Entropy \\
\hline
\end{tabular}

\section{TOPOLOGICAL CHARGE OF THE BLACK HOLE FROM HOLOGRAPHY}

One of the most exciting developments in modern theoretical physics is that the traditional black hole thermodynamics has acquired a new and interesting interpretation in AdS/CFT 7 [9]. In particular, this interpretation has been made systematic and universal in much more general settings of holography[10, 11].

Actually, Strominger and collaborators 12] first established hydrodynamics on a finite cutoff surface $r=r_{c}$ in the context of holographic gravity in 2011. It was later realized that this holographic correspondence on a finite cutoff surface, called a holographic screen, can be regarded as a natural extension of the AdS/CFT by a very general bulk/boundary holographic principle [8 11, 13].

Since we will focus on the black hole thermodynamics, here we only present this general holographic principle at finite temperature. This principle is just the correspondence between the Euclidean partition functional of the bulk black hole and the finite temperature generating functional (for a given ensemble) of the effective field theory on the holographic screen, namely

$$
Z_{F T}[J]=Z_{b u l k}[\bar{\phi}(=J)]
$$

where $J$ is the external source in the field theory, which is identified with the boundary value $\bar{\phi}$ of some bulk field(s) $\phi$. Taking logarithms of both sides of the above relation gives

$$
Z_{F T}^{c}[J] \approx I_{b u l k}^{o s}[\bar{\phi}]
$$

where $Z_{F T}^{c}[J]$ is the connected generating functional and we have used the semi-classical (or saddle point) approximation of the quantum gravity to reduce the bulk side down to the on-shell action of the classical gravity with fixed $\bar{\phi}$. Upon variation with respect to $J$, the above relation gives the connected Green function (correlation function) in the field theory in terms of variations of the bulk on-shell action with respect to $\bar{\phi}$, which is just the so-called holographic dictionary. In particular, this principle gives the ordinary AdS/CFT dictionary upon taking into account the holographic renormalization (see [13] for a review) to the otherwise divergent on-shell action for the holographic screen tending to the conformal boundary of an asymptotic AdS bulk space-time[11].

For our purpose, we only need to consider the one-point function, which reads from variation of (7) as

$$
\left\langle\mathcal{O}_{\phi}(x)\right\rangle_{F T}=\frac{\delta I_{\text {bulk }}^{\text {os }}[\bar{\phi}]}{\delta \bar{\phi}(x)}
$$

where the saddle point approximation is always assumed (with " $\approx$ " suppressed) and

$$
\mathcal{O}_{\phi}(x)=\frac{\delta I_{F T}[J]}{\delta J(x)}
$$

is the field variable (operator upon quantization) dual to the bulk field $\phi$. Note that the above expression is just formal, since in general the action $I_{F T}[J]$ of the boundary field theory cannot be explicitly written down.

Similar to the standard AdS/CFT, a bulk Maxwell field $A_{\mu}$ is dual to a global U(1) conserved current $j^{a}$ on the holographic screen, with the precise correspondence

$$
\left\langle j^{a}(x)\right\rangle=\frac{1}{\sqrt{g}} \frac{\delta I_{\text {bulk }}^{o s}[\bar{A}]}{\delta \bar{A}_{a}(x)}=-\left.n_{\mu} F^{\mu a}(x)\right|_{\text {bdry }}
$$

according to (8), where $n^{\mu}$ is the outward unit normal to the screen. The most important examples (including the above one) of the dictionary [10, 11] that we will use in the following are listed in Table 【.

Considering the holographic screen $r=r_{c}$ in the RN-AdS bulk space-time (11), one can easily show that the BrownYork tensor [14] takes the form of a (relativistic) perfect fluid, from which the energy density $\epsilon$ and pressure $p$ can be 
read off as

$$
\epsilon=C-\frac{d-1}{8 \pi G} \frac{\sqrt{f_{c}}}{r_{c}}, \quad p=\frac{d-2}{8 \pi G} \frac{\sqrt{f_{c}}}{r_{c}}+\frac{1}{16 \pi G} \frac{f_{c}^{\prime}}{\sqrt{f_{c}}}-C, \quad f_{c}:=f\left(r_{c}\right) .
$$

Here $C$ is a constant contributed from considerations of holographic renormalization[11], which is not important in the following discussion. Naturally defining the total volume 3

$$
V=\Omega_{d-1} r_{c}^{d-1}
$$

and energy

$$
E=\epsilon V
$$

one obtains the first law of thermodynamics

$$
d E+p d V=T d S+\mu d Q
$$

where the chemical potential

$$
\mu=-\frac{d-1}{8 \pi G} \frac{\Omega_{d-1} Q}{\sqrt{f_{c}}}\left(\frac{1}{r_{c}^{d-2}}-\frac{1}{r_{h}^{d-2}}\right)
$$

is proportional to the difference of electric potentials between the holographic screen and the horizont Note that here the temperature $T=T_{H} / \sqrt{f_{c}}$ is the local Hawking temperature on the screen, which differs from the Hawking temperature $T_{H}$ in (4) by a redshift factor. Actually, a thermodynamic relation similar to (13) has already been obtained in [14, 15] without referring to holography. Only with the principle (7) of holographic correspondence does the relation (13) have the meaning of thermodynamics for the dual field theory on the holographic screen. In more general gravitational theories (with more general matter content), one can see that this holographic first law (13) of thermodynamics still holds by using Hamilton-Jacobi-like analyses[11].

In the planar case $(k=0)$, since the dual field theory lives in an ordinary flat space-time $\left(r=r_{c}\right)$, it is expected that the Gibbs-Duhem relation

$$
E+p V=T S+\mu Q
$$

in the standard thermodynamics should hold, which can be straightforwardly verified and generally proved by extensibility (or scaling) arguments[11]. However, it is not the case for the non-planar configuration $(k \neq 0)$, which is also unsurprising because of the extra scale introduced by the spatial curvature of the screen. Actually, from the expressions (3), (10)-(12), (14), the condition $f\left(r_{h}\right)=0$ and

$$
T=\frac{T_{H}}{\sqrt{f_{c}}}=\frac{1}{2 \pi \sqrt{f_{c}}}\left[\frac{r_{h}}{\ell^{2}}+(d-2) \frac{M}{r_{h}^{d-1}}-(d-2) \frac{Q^{2}}{r_{h}^{2 d-3}}\right],
$$

after some straightforward arithmetics we have

$$
E+p V-T S-\mu Q=\frac{\Omega_{d-1} k}{8 \pi G \sqrt{f_{c}}}\left(r_{h}^{d-2}-r_{c}^{d-2}\right),
$$

which vanishes only if $k=0$ for $r_{c} \neq r_{h}$.

Most interestingly, it turns out that even in the non-planar case, the Gibbs-Duhem relation (15) can be rectified by introducing a new "charge"

$$
\varepsilon=\Omega_{d-1} k^{\frac{d-1}{2}}
$$

and its conjugate quantity

$$
\varsigma=\left(\frac{\partial E}{\partial \varepsilon}\right)_{S, Q, V}=\frac{\varepsilon^{\frac{3-d}{d-1}} \Omega_{d-1}^{\frac{d-3}{d-1}}}{8 \pi} \frac{r_{h}^{d-2}-r_{c}^{d-2}}{\sqrt{f_{c}}},
$$

\footnotetext{
${ }^{3}$ Note that the volume $\Omega_{d-1}^{(k)}$ depends on the compactifications for $k \leq 0$ as mentioned previously, but hereafter we assume that this volume is a constant $\Omega_{d-1}=\Omega_{d-1}^{(k=1)}$ under suitable compactifications (and rescaling of $k$ if necessary) [11].

${ }^{4}$ See the next section for a more precise description.
} 
which means that we have an extended first law

$$
d E+p d V=T d S+\mu d Q+\varsigma d \varepsilon .
$$

From (17), (18) and (19), one readily sees that the Gibbs-Duhem-like relation

$$
E+p V=T S+\mu Q+\varsigma \varepsilon
$$

still holds. Importantly, it can be shown that for odd $d$ this new "charge" $\varepsilon$ is proportional to the Euler number, i.e. a topological charge, while for even $d$ this interpretation is just formal[11] 5 Recall that in standard thermodynamics for every conserved charge we can introduce its conjugate potential, so it is really not strange that the topological charge $\varepsilon$ and its conjugate quantity $\varsigma$ play a role in the first law of thermodynamics for a system in a curved space (with non-trivial topology). In fact, this new charge $\varepsilon$ just takes into account the extra scale introduced by the spatial curvature, which makes the usual scaling arguments valid to produce the Gibbs-Duhem(-like) relation (21) 6 On the other hand, since the scaling arguments use essentially the property of thermodynamic quantities (e.g. the entropy $S$ ) as homogeneous functions of the complete set of independent extensive variables (basically conserved charges, except the volume $V$, for $S$ being the considered thermodynamic quantity) of the system, the Gibbs-Duhem(-like) relation just fails if we have not taken into account all the conserved charges. Therefore, the relation (21) may be thought of as a completeness relation, which means that we have known all the conserved charges of this holographic black hole system. In this sense, we say that $\varepsilon$ is the last charge of a black hole in the Einstein-Maxwell theory, which we have missed before.

Strictly speaking, the topological charge $\varepsilon$ should be an integer, as well as the electric charge $Q$ should be quantized, which makes the first law (20) less interesting. However, when this law is expressed in terms of densities:

$$
d \epsilon=T d s+\mu d \rho+\varsigma d e
$$

with the entropy density $s=S / V$, electric charge density $\rho=Q / V$ and topological charge density (Euler density) $e=\varepsilon / V$, the quantization of $\varepsilon$ and $Q$ can be smoothed out by a large volume $V$ of the boundary system. Actually, in the hydrodynamic regime of holography (the fluid/gravity duality) [16], the system can be inhomogeneous and (22) will hold locally. In this case, the Euler density $e$ and its conjugate potential $\varsigma$ are really dynamical quantities.

\section{TOPOLOGICAL CHARGE IN THE BLACK HOLE THERMODYNAMICS}

The existence and importance of the topological charge of black holes will be more convincing if it also plays some role in the (ordinary) black hole thermodynamics. Especially, things will be made more interesting by considering a slightly generalized framework of the standard black hole thermodynamics, which enables one to obtain a "thermodynamic" relation between off-horizon quantities of the black hole and which includes the standard black hole thermodynamics as a special (on-horizon) case.

Basically, there are two approaches to achieve this goal. The first approach is called "black hole thermodynamics on equipotential surfaces", which is proposed in [17]. Considering variation between different configurations of the RN-AdS black hole (11) related to an equipotential surface $f(r)=c$ with fixed $c$, one easily obtains the generalized first law

$$
d \tilde{M}=T d S+\Phi d Q
$$

with $\tilde{M}$ the same ADM mass as in (5),

$$
T(r)=\frac{f^{\prime}(r)}{4 \pi}
$$

the Unruh-Verlinde temperature 17, 19, 20],

$$
S(r)=\frac{\Omega_{d-1} r^{d-1}}{4}
$$

\footnotetext{
${ }^{5}$ Note that if $\varepsilon$ is not defined by (18), then the relation (21) and the first law (20) cannot hold simultaneously, so the validity of the Gibbs-Duhem-like relation can be viewed as equivalent to the fact that $\varepsilon$ is the topological charge.

${ }^{6}$ In other words, the effect of the non-extensibility caused by the spatial curvature can be properly accounted for, at least in the thermodynamics under consideration, by the introduction of the extra charge $\varepsilon$.
} 
the Wald-Padmanabhan entropy 7 and

$$
\Phi(r) \sim \frac{Q}{r^{d-2}}
$$

the electric potential at the equipotential surface. When $c=0$, i.e. $r=r_{h}$, the equipotential surface is just the horizon and the above first law becomes that of the standard black hole thermodynamics. This formalism has been generalized to arbitrary dimensions and the general Lovelock-Maxwell theory in [19, 20].

Now we illustrate how to incorporate the topological charge into the above formalism. Writing the gravitational potential $f(r)$ in (11) as

$$
f(r, k, M, Q)=k+\frac{r^{2}}{\ell^{2}}-\frac{2 M}{r^{d-2}}+\frac{Q^{2}}{r^{2 d-4}},
$$

we have for the equipotential surface $f=c$ (constant)

$$
d f(r, k, M, Q)=\frac{\partial f}{\partial r} d r+\frac{\partial f}{\partial k} d k+\frac{\partial f}{\partial M} d M+\frac{\partial f}{\partial Q} d Q=0 .
$$

Noting

$$
\begin{aligned}
& \partial_{r} f=4 \pi T, \quad \partial_{k} f=1, \\
& \partial_{M} f=-\frac{2}{r^{d-2}}, \quad \partial_{Q} f=\frac{2 Q}{r^{2 d-4}},
\end{aligned}
$$

we obtain

$$
d M=2 \pi \operatorname{Tr}^{d-2} d r+\frac{r^{d-2}}{2} d k+\frac{Q}{r^{d-2}} d Q
$$

Defining the entropy $S$ as in (24), we finially see the generalized first law

$$
d \tilde{M}=T d S+\varpi d \varepsilon+\Phi d Q, \quad \varpi:=\frac{\varepsilon^{\frac{3-d}{d-1}} \Omega_{d-1}^{\frac{-2}{d-1}}}{2 \pi(d-1)} S^{\prime},
$$

where $S^{\prime}:=d S / d r, \Phi$ is given by (25) and $\varepsilon$ is exactly the topological charge (18). Remarkably, its appearance in the above first law is not artificial, since the conjugate quantity (19) in the holographic thermodynamics is just the difference of the above conjugate potential $\varpi$ (called the topological potential) between the holographic screen (as the equipotential surface) and the horizon, redshifted by $\sqrt{f_{c}}$. Actually, as briefly mentioned in the previous section, the structure for the electric charge is similar [10, 11]: The chemical potential $\mu$ (conjugate to $Q$ ) in the holographic thermodynamics is just the difference of the above electric potential $\Phi$ between the holographic screen and the horizon, redshifted by $\sqrt{f_{c}}$.

The above black hole thermodynamics with the topological charge can be easily generalized to the Lovelock-Maxwell theory [11], where the entropy $S$ becomes the Wald-Padmanabhan entropy

$$
S=\frac{d-1}{4} \Omega_{d-1} r^{d-1} \sum_{j} \frac{j \tilde{\alpha}_{j}}{d-2 j+1}\left(\frac{k-f}{r^{2}}\right)^{j-1}
$$

and the topological potential is still given by (27) but now $S^{\prime}$ is defined as

$$
S^{\prime}:=\frac{d}{d r} S(r, f(r))
$$

Noting that in the Lovelock-Maxwell case the entropy (28) depends nontrivially on $k$, we again emphasize that the appearance of $\varepsilon$ in the generalized first law (27) is not tuned by hand.

The second approach is called "black hole thermodynamics from gravitational equations of motion". In this approach, we just put the metric of the maximally symmetric black holes into Einstein's equations (or more generally the

\footnotetext{
7 This entropy is the standard Wald entropy generalized to the off-horizon case by Padmanabhan 18], which is proportional to the area of the equipotential surface for the Einstein gravity. See also [19, 20].
} 
equations of motion of the Lovelock gravity) with matter, which is then recognized as the first law of thermodynamics with the topological charge. For simplicity, we take the metric ansatz

$$
d s_{d+1}^{2}=\frac{d r^{2}}{f(r)}-f(r) d t^{2}+r^{2} d \Omega_{d-1}^{(k) 2}
$$

which is enough for the Einstein-Maxwell (or Lovelock-Maxwell) case that we focus on in this essay. The most general case of a maximally symmetric black hole can also be treated similar to the case without the topological charge in [20]. Upon substitution of the above ansatz into Einstein's equations

$$
R_{\mu \nu}-\frac{1}{2} R g_{\mu \nu}=8 \pi T_{\mu \nu}
$$

with $T_{\mu \nu}$ the stress-energy tensor of the matter, the nontrivial part of them is

$$
r f^{\prime}-(d-2)(k-f)=\frac{16 \pi P}{d-1} r^{2}
$$

with $P=T_{r}^{r}=T_{t}^{t}$ the radial pressure of the matter. Now we focus on a maximally symmetric screen with fixed $f$ in different static, maximally symmetric solutions of (30). In order to do so, we just need to compare two such configurations of infinitesimal difference. In fact, multiplying both sides of (31) by the factor

$$
\frac{d-1}{16 \pi} \Omega_{d-1} r^{d-3} d r
$$

we have after some simple algebra (assuming $f$ fixed)

$$
\frac{f^{\prime}}{4 \pi} d\left(\frac{\Omega_{d-1} r^{d-1}}{4}\right)-d\left(\frac{d-1}{16 \pi} \Omega_{d-1}(k-f) r^{d-2}\right)+\frac{d-1}{16 \pi} \Omega_{d-1} r^{d-2} d k=P d\left(\frac{\Omega_{d-1} r^{d}}{d}\right) .
$$

The above equation is immediately recognized as the first law

$$
T d S-d E+\varpi d \varepsilon=P d \mathcal{V}
$$

with $T$ again the Unruh-Verlinde temperature (23) on the screen, $S$ again the Wald-Padmanabhan entropy (24) of the screen, $\varpi$ the same topological potential as in (27), $\varepsilon$ again the topological charge (18), $\mathcal{V}=\Omega_{d-1} r^{d} / d$ the volume of the (standard) $d$-"ball" and

$$
E=\frac{d-1}{16 \pi} \Omega_{d-1}(k-f) r^{d-2}
$$

the Misner-Sharp energy inside the screen[21].

Then we generalize the above discussion to the Lovelock gravity. In this case, the nontrivial part of the equations of motion is

$$
\sum_{j} \tilde{\alpha}_{j}\left(\frac{k-f}{r^{2}}\right)^{j-1}\left[j r f^{\prime}-(d-2 j)(k-f)\right]=\frac{16 \pi P}{d-1} r^{2} .
$$

Following the same logic as in the Einstein gravity, after multiplying both sides of the above equation by the same factor (32) we obtain

$$
\begin{aligned}
& \frac{f^{\prime}}{4 \pi} d\left(\frac{d-1}{4} \Omega_{d-1} r^{d-1} \sum_{j} \frac{j \tilde{\alpha}_{j}}{d-2 j+1}\left(\frac{k-f}{r^{2}}\right)^{j-1}\right) \\
& -d\left(\frac{d-1}{16 \pi} \Omega_{d-1} r^{d} \sum_{j} \tilde{\alpha}_{j}\left(\frac{k-f}{r^{2}}\right)^{j}\right)+\frac{d-1}{16 \pi} \Omega_{d-1} \sum_{j} j \tilde{\alpha}_{j}(k-f)^{j-1} r^{d-2 j} d k \\
= & P d\left(\frac{\Omega_{d-1} r^{d}}{d}\right) .
\end{aligned}
$$

The above equation is again recognized as the first law (33) with the Wald-Padmanabhan entropy (28) in the Lovelock gravity and (29) in the definition (27) of the topological potential. 


\section{CONCLUSION AND DISCUSSION}

To conclude, we have shown in this essay that a maximally symmetric black hole has a topological charge $\varepsilon$, besides the usual conserved charges (the mass $M$ and electric charge $Q$ ) and the Noether charge $S$. This topological charge has been largely neglected, if not totally missed, before, but it naturally arises in the holography with a maximally symmetric screen. In the sense of the Gibbs-Duhem-like relation (21) viewed as a completeness relation, this topological charge is the last charge of the maximally symmetric black hole. The existence of this charge and the interesting role played by it has then been shown in the black hole thermodynamics from two different approaches, as general as in the Lovelock(-Maxwell) theory.

In the context of AdS/CFT, actually, there are already discussions about the deviation from the Gibbs-Duhem relation (15) in the case of $k=1$, which is related to the Casimir energy by the Cardy-Verlinde formula (see, for example, [22, 23] and references therein). However, it seems not clear the precise relation between those discussions and the topological charge introduced here.

The topological charge with its associated first law of thermodynamics has some direct implications/applications. The first one is to consider how the extended thermodynamics changes the thermodynamic behavior of black holes. Actually, there is already some progress along this direction[24]. The second one is to investigate its application in the the fluid/gravity duality, as mentioned at the end of Sec. II where the holographic screen can have a curved geometry determined by dynamics, as long as its curvature variation is temporally and spatially slow enough to stay within the hydrodynamic regime.

It is also interesting to ask whether more general black holes, not only the maximally symmetric ones, have topological charges. Perhaps the simplest non-maximally symmetric black holes are those with horizons being direct products of maximally symmetric spaces, but it is not clear whether holography can be well defined in presence of such bulk black holes. A more interesting case is a rotating black hole, i.e. Kerr(-AdS) black hole in Einstein's gravity. In this case, it turns out that a first-law-like relation including the topological charge can be written down, but a completeness check similar to the Gibbs-Duhem-like relation (21) is still lacking, again due to the unclear holography for a black hole with angular momentum. The discussion of the rotating case is also limited by the fact that up to now we have no analytical solution of such black holes in the Lovelock(-Maxwell) theory. Anyway, the topological charge of black holes beyond the maximally symmetric case is worthy of further investigation.

\section{ACKNOWLEDGMENTS}

The author would like to thank Xiaoning Wu, Hongbao Zhang and Liming Cao for helpful discussions or valuable comments. He also thanks the Yukawa Institute for Theoretical Physics at Kyoto University, where this paper was pushed forward during the workshop YITP-T-17-02 "Gravity and Cosmology 2018". This work is partly supported by the National Natural Science Foundation of China (Grant Nos. 11475179 and 11675015). It is also supported by the "Strategic Priority Research Program of the Chinese Academy of Sciences", grant No. XDB23030000.

[1] D. Kastor, S. Ray and J. Traschen, Class. Quant. Grav. 26 (2009) 195011 arXiv:0904.2765.

[2] B.P. Dolan, Class. Quant. Grav. 28 (2011) 235017 arXiv:1106.6260.

[3] M.C. Baldiotti, R. Fresneda and C. Molina, Ann. Phys. 382 (2017) 22 arXiv:1701.01119.

[4] D. Kastor, S. Ray and J. Traschen, Class. Quant. Grav. 27 (2010) 235014 arXiv:1005.5053.

[5] C. Teitelboim, Phys. Lett. B 158 (1985) 293.

[6] R.M. Wald, Phys. Rev. D 48 (1993) 3427 gr-qc/9307038.

[7] J. Maldacena, Adv. Theor. Math. Phys. 2 (1998) 231 hep-th/9711200.

[8] S.S. Gubser, I.R. Klebanov and A.M. Polyakov, Phys. Lett. B 428 (1998) 105 hep-th/9802109.

[9] E. Witten, Adv. Theor. Math. Phys 2 (1998) 253 hep-th/9802150.

[10] Y. Tian, X.-N. Wu and H. Zhang, Class. Quant. Grav. 30 (2013) 125010 arXiv:1204.2029.

[11] Y. Tian, X.-N. Wu and H. Zhang, JHEP 1410 (2014) 170 arXiv:1407.8273.

[12] I. Bredberg, C. Keeler, V. Lysov and A. Strominger, From Navier-Stokes To Einstein, arXiv:1101.2451.

[13] K. Skenderis, Class. Quant. Grav. 19 (2002) 5849 hep-th/0209067].

[14] J.D. Brown and J.W. York, Phys. Rev. D 47 (1993) 1407.

[15] J.D. Brown and J.W. York, Phys. Rev. D 47 (1993) 1420.

[16] S. Bhattacharyya, V.E. Hubeny, S. Minwalla and M. Rangamani, JHEP 0802 (2008) 045 arXiv:0712.2456.

[17] Y.-X. Chen and J.-L. Li, Phys. Lett. B 700 (2011) 380 arXiv:1006.1442.

[18] T. Padmanabhan, Phys. Rev. D 81 (2010) 124040 arXiv:1003.5665.

[19] Y. Tian and X.-N. Wu, Phys. Rev. D 83 (2011) 021501 arXiv:1007.4331. 
[20] Y. Tian and X.-N. Wu, JHEP 1101 (2011) 150 arXiv:1012.0411.

[21] C.W. Misner and D.H. Sharp, Phys. Rev. 136 (1964) B571.

[22] R.-G. Cai, L.-M. Cao and D.-W. Pang, Phys. Rev. D 72 (2005) 044009 hep-th/0505133.

[23] G.W. Gibbons, M.J. Perry and C.N. Pope, Phys. Rev. D 72 (2005) 084028 hep-th/0506233].

[24] S.-Q. Lan, Advances in High Energy Physics (2018) 4350287 arXiv:1805.01326]. 